\title{
Il diritto delle comunità umane intenzionali: nuovi ordinamenti giuridici?
}

\section{Nozione di comunità}

$\|$ sostantivo 'comunità', deriva dal termine latino communis, ed oltre ad appartenere al linguaggio di uso corrente, il suo impiego si riscontra anche nella terminologia tecnica di discipline differenti: con significati di non facile esplicitazione è utilizzato essenzialmente nell'antropologia e nella sociologia, ma anche nella filosofia, nel diritto, e nella scienza politica ${ }^{1}$.

Nell'ambito delle scienze sociali il sostantivo comunità è principalmente impiegato con due accezioni.

Nella sociologia classica, infatti, il termine comunità sta ad indicare una tipologia peculiare di rapporti sociali che coinvolgono totalmente l'individuo di una collettività organizzata: pertanto, il sostantivo, in questa ampia accezione significante, racchiude in sé sia le piccole comunità di villaggio e sia la più ampia comunità nazionale, ricomprendendo entità come la famiglia, ma anche qualsiasi altro nucleo sociale in situazioni di elevata integrazione, fino a delineare la peculiare forma della società tradizionale antecedente a quella attuale.

Nella sociologia contemporanea, invece, il significato di comunità coincide con quello di comunità locale.

Il concetto di comunità, pertanto, è stato ben delineato nell'ambito delle scienze sociali, in cui spiccano le teorie di alcuni importanti studiosi rappresentanti sia della sociologia classica quali Ferdinand Tönnies ${ }^{2}$ (il quale enuclea

\footnotetext{
* Il dR. Salvatore Piliu, è attualmente dottorando di ricerca presso la Facultè de Droit de l'Université di Rouen - Normandie, France, e sta conducendo una ricerca sul tema: Analyse structurale et qualification juridique des communautés intentionnelles: les cas de Damanhur, Les Amannins et Arcosanti entre Sociologie juridique et Droit comparé. Email: salvadorfelice@yahoo.it

${ }^{1}$ Cfr. A. Bagnasco, Voce "Comunità", in Enciclopedia Treccani delle Scienze sociali, 1992, $1 \mathrm{~s}$.

2 F. Tönnies, Comunità e società, (tit. orig.: Gemeinschaft und Gesellshaft), Roma 2011, 47 ss.
} 
il concetto di comunità-società, Gemeinschaft-Gesellschaft, come mezzo basilare per cogliere il mutamento sociale. Per l'Autore la comunità deve essere recepita "come un organismo vivente", mentre la società deve intendersi "come un aggregato e prodotto meccanico"), o Max Weber (per il quale la nozione di comunità è situata al livello delle relazioni sociali, ovvero per l'Autore, una relazione sociale può essere definita come comunità "se, e nella misura in cui, la disposizione dell'agire sociale poggia [...] su una comune appartenenza, soggettivamente sentita (affettiva o tradizionale) dagli individui che ad essa partecipano") ${ }^{3}$, sia della sociologia contemporanea, come Talcott Parsons ${ }^{4}$, il quale, usa il concetto di comunità solo per individuare quella tipologia di collettività "i cui membri condividono un'area territoriale come base di operazioni per le attività giornaliere".

Parsons inoltre enuclea il concetto "comunità societaria" che ha come finalità l'integrazione; in tale sottosistema l'Autore individua degli "obblighi di lealtà nei confronti della collettività societaria, sia per il complesso dei suoi membri, che per le varie categorie, diversificate per il loro status e ruolo, che si ritrovano all'interno della società" .

Appare evidente come l'uso del termine comunità sia sicuramente problematico, non solo per il fatto evidente che nella stessa parola si sovrappongono accezioni differenti, ma più in generale per difficoltà di precisazione concettuale e scarsa capacità della ricerca.

Il problema della nozione di comunità ha comunque assunto nuova rilevanza, se rapportata sia alla decadenza del concetto di Stato, inteso in senso tradizionale, sia alle procedure di globalizzazione.

Come giustamente afferma Z. Bauman, "oggi la comunità è considerata e ricercata come un riparo dalle maree montanti della turbolenza globale, maree originate di norma in luoghi remoti che nessuna località può controllare in prima persona"'.

Un ulteriore e rapido richiamo etimologico, però, a mio avviso, può essere adeguato al fine di motivare e comprendere l'evoluzione delle diverse linee di pensiero sopra indicate.

Il termine comunità, come già affermato in apertura di questo contributo, può essere riconducibile al concetto di communitas ed anche di koinonia. Nella parola koinonia, i concetti di denotazione e connotazione confluiscono nel significato di una unione (koinè), in cui il singolo non ha un'esistenza autonoma da ciò

\footnotetext{
${ }^{3}$ M. Weber, Wirtschaft und Gesellschaft, Tübingen 1922 (tr. it.: Economia e società, 4 voll., Milano 1961), 23 ss.

${ }_{4}$ T. Parsons, The social system, Glencoe, Ill., 1951 (tr. it.: Il sistema sociale, Milano 1965), 97 ss.

${ }_{5}^{5}$ T. Parsons, The system of modern societies, Englewood Cliffs, N. J., 1971 (tr. it.: Sistemi di società, Bologna 1973), 28 ss.

${ }^{6}$ Z. Bauman, Voglia di comunità, Roma-Bari, 2001, 138 ss.
} 
che la comunità raffigura e rappresenta: la sua sorte è delineata entro i confini dalla comunità di appartenenza. Il termine communitas, invece, può avere una accezione differente rispetto a koinonia se si evidenzia il concetto di munus, nel suo collegamento al prefisso cum. Infatti, se si sottolinea come munus stia ad indicare 'unire', il significato di comunità non si attaglierà nell'appartenenza identitaria, quanto nella corrispondenza biunivoca dell'obbligo donativo; il vincolo comunitario, pertanto, coincide con un rapporto 'dare-darsi'”.

\section{Nozione di comunità intenzionale}

In seno alla nozione di comunità si inserisce la concezione più specifica di 'Comunità Intenzionale', che va a definire un fenomeno globale che racchiude in sé differenti realtà, come le comunità intenzionali ecosostenibili (o ecoreversibili), gli ecovillaggi, il cohousing e i condomini solidali.

Per la Fellowship for Intentional Communities -FIC- una Comunità Intenzionale rappresenta "un gruppo di persone che hanno scelto di lavorare insieme con l'obiettivo di un ideale o una visione comune", e vi rientrano senza alcun dubbio gli ecovillaggi, che rappresentano una realtà sempre più diffusa nel mondo ${ }^{8}$.

Vista la molteplicità di esperienze, è difficile tracciarne un profilo che possa esaustivamente appartenere a tutti i progetti comunitari. Rimanendo pertanto in termini generici, è possibile però affermare che nelle Comunità Intenzionali sono presenti tre aspetti tra loro sinergici:

1) la condivisione di uno stesso progetto di vita, che spesso ha riflessi anche in ambito lavorativo;

2) un'organizzazione interna, in grado di sviluppare meccanismi solidaristici e di aiuto reciproco;

3) l'adozione di stili di vita sostenibili, che consentono un ridotto impatto ambientale.

Si potrebbe pertanto raffigurare l'idea di comunità intenzionale con quella di una grande famiglia allargata, dove ciò che conta non sono i vincoli di parentela ma l'intenzione, la volontà delle persone che ne fanno parte di creare uno spazio di vita fuori dalle logiche di competizione e sopraffazione.

Pertanto, l'elemento fondante di ogni comunità intenzionale è la condivisione di idee, passioni, visioni del mondo ma anche spazi, beni di vario genere e denaro. Sono differenti i livelli di condivisione praticati dai membri delle

\footnotetext{
A. Bagnasco, Voce "Comunità", 1 ss.

${ }^{8}$ Cfr. M. Olivares, Comuni, comunitá ed ecovillaggi, London, 2010, 5 ss.
} 
comunità intenzionali. Vi sono ad esempio ecovillaggi in cui predomina l'elemento propriamente comunitario e dove non viene riconosciuto quasi nessun tipo di proprietà privata, dove terreni, abitazioni, mezzi di produzione e di locomozione, così come le riserve valutarie, appartengono alla comunità. Ve ne sono altri - con un orientamento più individualista - in cui proprietà privata e proprietà collettiva svolgono funzioni diverse e complementari ${ }^{9}$.

\section{Comunità intenzionali e diritto}

Con queste premesse, in assenza di una specifica disciplina di riferimento che possa regolarne le attività, non è raro che nella vita delle Comunità Intenzionali insorgano problematiche di varia natura, come ad esempio in relazione alla proprietà degli immobili, all'organizzazione del lavoro ed alle soluzioni urbanistiche a misura di gruppi, che hanno esigenze di spazio e di organizzazione diverse da quelle di una famiglia.

A tale proposito, attualmente, in Italia si è aperto un dibattito politico in merito al riconoscimento giuridico delle comunità intenzionali, che è sfociato nell'elaborazione di un progetto di legge appena presentato in Parlamento ${ }^{10}: \mathrm{i}$ temi principali affrontati nel suddetto Progetto di legge riguardano la definizione di comunità intenzionale; i diritti e i doveri verso i membri della propria comunità; la possibile attribuzione o gestione da parte delle comunità di proprietà demaniali rurali e liminali; il regime fiscale e lavorativo e il bilancio etico-sociale.

Le Comunità Intenzionali però regolano i rapporti intersoggettivi al loro interno con disposizioni che hanno un autonomo valore giuridico e che vanno a disciplinare, per gli appartenenti alla comunità, sia gli ambiti del diritto privato, in particolare le problematiche inerenti alla proprietà dei beni, (spesso come già sottolineato in precedenza si configura l'istituto giuridico della proprietà collettiva), i rapporti nell'ambito familiare; sia quelli del diritto pubblico, con l'emanazione di Carte Costituzionali che racchiudono norme con valenza pubblica: si tratta pertanto di veri e propri ordinamenti giuridici.

\section{Comunità intenzionali e diritto: il caso di Damanhur}

Nell'ambito della mia attuale ricerca sulle Comunità intenzionali, ho avuto modo di focalizzare la mia attenzione su tre comunità intenzionali nel mondo: Damanhur in Italia, Les Amanins in Francia, Arcosanti negli Stati Uniti

\footnotetext{
${ }^{9}$ Cfr. M. Olivares, Comuni, cit., 5 ss.

${ }^{10}$ Proposta dell'On. Mirko Busco, in discussione al Parlamento italiano il 7 giugno 2017.
} 
d'America. Appare evidente, come ad es. nel caso di Damanhur ${ }^{11}$, situata in Piemonte, che si tratta di una Comunità Intenzionale con una struttura formale e giuridica ben definita, con un territorio vasto per estensione e un numero di persone residenti abbastanza alto: una collettività organizzata nel vero e proprio senso della parola.

A Damanhur esiste una Costituzione redatta in articoli che racchiude in sé i diritti fondamentali dei residenti, nonché una serie di norme ben congeniate che disciplinano i rapporti giuridici di natura privatistica e pubblicistica. La nuova carta costituzionale di Damanhur è stata redatta nel 2007, si compone di 15 articoli ed è andata a sostituire la precedente Costituzione del 1981, che si sviluppava il 130 articoli.

Dalla lettura delle norme appare evidente un'impronta di forte ispirazione etica che va a connotare la Carta costituzionale per quanto attiene il profilo morale dei consociati (art. 1-8). Ma dal punto di vista giuridico appaiono di grande rilevanza alcune norme che attengono alla organizzazione politico sociale della Comunità che si presenta come una Federazione di Comunità, in cui è possibile ottenere la cittadinanza (art. 10). Vi sono norme che riguardano la maggiore carica direttiva delle comunità che è affidata al Re Guida eletto fra i consociati con carica temporanea (art. 11) e vi è anche un Collegio di Giustizia (art. 12) che è preposto al controllo sul rispetto delle norme dei consociati ed assume anche competenze giurisdizionali. Vi sono anche norme in merito ai rapporti di diritto privato (proprietà e conferimenti di tipo economico finanziario, e organizzazione familiare, artt. 8 e 9). In poche parole, una collettività organizzata con norme e poteri legislativi disciplinati ad hoc (artt. 13-15) ${ }^{12}$.

Anche in questo caso ci troviamo in un contesto dove vale la massima latina ubi societas, ibi ius. Ma è interessante notare come Damanhur venga definita dai residenti come un laboratorio per il futuro dell'umanità, dove il pensare al bene degli altri, la spinta alla continua trasformazione interiore, la solidarietà e il rispetto tra i cittadini, il dare sempre agli altri un'ulteriore possibilità di

${ }^{11}$ J. Merrifield, Damanhur, The Story of an Extraordinary Italian Artistic and Spiritual Community, London, 2006. Nello specifico Damanhur è una Federazione di Comunità spirituali sita a nord del Piemonte, tra Torino e Aosta, in un raggio di 15 chilometri che comprende al centro la Valchiusella, una valle ancora verde e pulita in cui i seicento cittadini di Damanhur che vi abitano hanno dato vita a una società multilingue, aperta agli scambi con il mondo e le diverse culture dei popoli. E' stata fondata nel 1975 su ispirazione di Falco Tarassaco, Oberto Airaudi (1950-2013). La sua visione illuminata e pragmatica ha creato una fertile realtà fondata su solidarietà, condivisione, amore reciproco e rispetto per l'ambiente, tanto da ottenere nel 2005 il riconoscimento come modello di società sostenibile dal Global Human Settlements Forum delle Nazioni Unite (ONU).

${ }^{12}$ R. Sparagio, I segreti del sistema politico di Damanhur. Agorà comunitarie ed elisir di lunga vita sociale, Vidracco, 2017, 6 ss. 
rilancio, la cura per l'ambiente e per tutte le forme di vita appaiono come principi fondamentali della Comunità.

Da queste mie ultime considerazioni, propongo un quesito conclusivo aperto, per il momento senza risposta, che invita ad una riflessione più ampia: le comunità intenzionali possono rappresentare l'alba di nuove società umane, ovvero possono rappresentare un nuovo modello di organizzazione sociale alternativo, fondato su modelli sociali più equi, partecipativi e solidali nel rispetto dei territori e dell'ambiente? Un interrogativo che mi propongo di risolvere nell'ambito della mia ricerca che a breve confluirà in una pubblicazione monografica.

\section{Bibliografia}

Airaudi O., Sincronicità e linee sincroniche, Torino, 1981.

Albert M., Il libro dell'economia partecipativa. La vita dopo il capitalismo, Milano, 2006.

Bagnasco A., Voce "Comunità", in Enciclopedia Treccani delle Scienze sociali, 1992.

Bauman Z., Voglia di comunità, Roma-Bari, 2001.

Bauman Z., La società dell'incertezza, Bologna, 2014, $150 \mathrm{p}$.

Bauman Z., Il demone della paura, Roma-Bari, 2014, 136 p.

Bauman Z., La solitudine del cittadino globale, Milano, 2014, 227 p.

Berzano L., Damanhur. Popolo e comunità, Torino 1998.

Brighenti A., Realmente distinti, ma inseparabili: il diritto e l'altro, in Sociologia del diritto, n. 2, 2003.

Carbonnier J., Sociologia giuridica, Torino, 2012, 399 p.

Carbonnier J., Sociologie juridique, Paris, 2004, 415 p.

Clark T.N., a cura di, Community structure and decisionmaking. Comparative analysis, San Francisco, 1968.

Cover R., Nomos e narrazione. Una concezione ebraica del diritto. A cura di Marco Goldoni, Torino, 2008, 145 p.

Cuche D., La notion de culture dans les sciences sociales, Paris, 2010, 157 p.

Deumier P., Le droit spontané, Paris, 2002, 477 p.

Deumier P., Introduction générale au droit, II éd., Paris 2013, 357 p.

Doytcheva M., Le multiculturalisme, Paris, 2011, 125 p.

Duguit L., Les transformations générales du droit privé depuis le Code Napoléon, Paris, 1912, 224 p.

Esposito R., Communitas. Origine e destino della comunità, Torino 1998.

Kymlicka W., Liberalism, community, and culture, Oxford, 1991.

Le Breton D., Fuggire da sé, Milano, 2016, 195 p.

Lynch O.M., a cura di, Culture and Community in Europe, New Delhi, 1984.

Macdonald R., L'hypothèse du pluralisme juridique dans les sociétés démocratiques avancées, Revue de droit de l'Université de Sherbrooke, n. 3, 2003.

Merrifield J., Damanhur: The Real Dream, London, 1998.

Merrifield J., Damanhur, The Story of an Extraordinary Italian Artistic and Spiritual Community, London, 2006.

Metcalf B., Damanhur: un magico viaggio iniziatico, Communities Magazine, n. 103, 1999.

Olivares M., Comuni, comunitá ed ecovillaggi, London, 2010, 242 p.

Palombo S., La mia Damanhur. La più grande comunità spirituale italiana raccontata da chi ci vive, Vidracco, 2011, $132 \mathrm{p}$.

Palombo S., Tempio nel verde. Cos'è e cosa rappresenta il Tempio Bosco Sacro, Vidracco, 2017, 98 p. 
Palombo S., Calati (F.), Viaggio nei templi dell'umanità. La straordinaria opera sotterranea dedicata alla spiritualità, Vidracco, 2015, $154 \mathrm{p}$.

Parisot V., Le droit spontaneè, Paris 2002, 477 p.

Parisot V., Les conflits internes de lois, Paris, 2013, 3062 p.

Parsons T., The social system, Glencoe, Ill., 1951 (tr. it.: Il sistema sociale, Milano 1965).

Parsons T., The system of modern societies, Englewood Cliffs, N. J., 1971 (tr. it.: Sistemi di società, Bologna 1973).

Pessina Dassonville S. (dir.), Le statut des peuples autochtones. À la croisée des savoirs, Paris 2012, 382 p.

Remotti F., Temi di antropologia giuridica, Torino 1982.

Romano S., L'ordinamento giuridico, Firenze 1946.

Romano S., L'ordre juridique, Dalloz 1975.

Rossi P., Il concetto di cultura, Torino 1970.

Rouland N., Antropologia giuridica, Milano 1992, 487 p.

Sacco R., Antropologia giuridica. Contributo ad una macrostoria del diritto, Bologna, 2007, 352 p.

Sacco R., Anthropologie juridique. Apport à une macro-historie du droit, Paris, 2008, 270 p.

Sacco R., Il diritto muto. Neuroscienze, conoscenza tacita, valori condivisi, Bologna, 2015.

Sartori G., Logica, metodo e linguaggio nelle scienze sociali, Bologna, 2011, 288 p.

Savidan P., Le multiculturalisme, Paris, 2012, 127 p.

Schultz E.A., Lavenda R.H., Antropologia culturale, (tit. orig. Cultural Anthropology: A Perpesctive on the Human Condition, VII ed., Oxford 2010), Bologna, 2010, 404 p.

Sparagio R., I segreti del sistema politico di Damanhur. Agorà comunitarie ed elisir di lunga vita sociale, Vidracco 2017, 94 p.

Taylor C., Le multiculturalisme. Différence et démocratie, Paris, 2009, 139 p.

Tonicelli A., Palombo S., Visita a Damanhur. Vita quotidiana, pensiero, storia di un popolo capace di sognare, Vidracco, 2016, $144 \mathrm{p}$.

Tönnies F., Comunità e società, (tit. orig.: Gemeinschaft und Gesellshaft) Roma 2011, 285 p.

Treves R., Sociologia del diritto, Torino 1988, 354 p.

Watson A., Evoluzione sociale e mutamenti del diritto, (tit. orig.: Society and Legal Change), Milano, 2006, 221 p.

Warner W. L., Lunt, P. S., The social life of a modern community, New Haven 1941.

Warner W. L., Lunt, P. S., The status system of a modern community, New Haven 1942.

Weber M., Wirtschaft und Gesellschaft, Tübingen 1922 (tr. it.: Economia e società, 4 voll., Milano 1961)

\section{Summary}

Il presente intervento si propone di analizzare dal punto di vista giuridico il nuovo fenomeno, oramai globalizzato, delle comunità umane intenzionali, con l'intento di comprendere se queste nuove tipologie di aggregazione di individui possano essere considerate come forme alternative di collettività organizzate produttive di modelli giuridici differenti rispetto a quelli tradizionali, ovvero capaci di dar vita a nuovi ordinamenti giuridici, partendo dall'assunto ubi societas, ibi ius. Partendo dalle definizioni elaborate nel campo della sociologia giuridica di comunità e di comunità intenzionali, si è poi analizzata la problematica inerente la capacità delle comunità intenzionali di produrre regole giuridiche da applicare ai componenti delle comunità. Ė stata poi esaminata l'organizzazione giuridica della Comunità di Damanhur, situata in Piemonte (Italia), in cui vi sono norme interne di diritto privato e di diritto pubblico che regolano la vita dei consociati, un vero e proprio sistema giuridico interno della Comunità, che pone non pochi problemi nel rapporto con il diritto italiano. Il nuovo progetto di legge presentato nel 2017 al Parlamento italiano, in merito al riconoscimento giuridico delle Comunità intenzionali, potrebbe regolare in maniera definitiva 
questa materia, ovvero i rapporti tra Comunità e Stato di appartenenza, con il conseguente riconoscimento della esistenza di 'ordinamenti giuridici autonomi' facenti capo alle Comunità intenzionali.

Keywords: Comunità; comunità intenzionali; diritto; nuovi ordinamenti giuridici.

\section{The law of international human communities a new legal order?}

\section{Summary}

The purpose of this paper is to analyze from a legal standpoint the new, globalized phenomenon of intentional human communities, in order to understand whether these new types of human collectives can be considered as alternative forms of traditional organizational models and capable of creating new legal systems, starting from the assertion ubi societas ibi ius. Basing on the definitions elaborated in the field of legal sociology and intentional communities, we analyzed a intentional community's capacity to produce legal rules that apply to that members. The legal organization of the Community of Damanhur, located in Piedmont (Italy), where there are internal rules of private and public law regulating the life of its members, is a genuine legal system, which poses many problems in relation to Italian law. The new bill submitted to the Italian Parliament in 2017 on the legal recognition of intentional communities could definitively regulate the relationship between an Intentional Community and the State, with the consequent recognition of the existence of 'autonomous legal systems' for all Intentional Communities.

Keywords: Community; Intentional communities; Law; New legal systems. 